SCIENTIFIC LETTER

\title{
p53Arg72Pro polymorphism of tumour suppressor protein is associated with luminal narrowing after coronary stent placement
}

\author{
S Kojima, N Iwai, N Tago, K Ono, K Ohmi, G Tsujimoto, S Takagi, S Miyazaki, H Nonogi, Y Goto
}

Heart 2004;90:1069-1070. doi: 10.1136/hrt.2002.007047

$\mathrm{T}$ he cause of in-stent luminal narrowing has been primarily considered to be neointimal hyperplasia that is caused by proliferating vascular smooth muscle cells (VSMC). It has been recently reported that local drug delivery systems produce good results for the inhibition of VSMC proliferation. ${ }^{1}$ The potential of suppressive agents in the treatment of in-stent luminal narrowing arises from basic studies according to cell cycle regulation and gene expression.

p53 is a tumour suppressor protein involved in regulating the growth of VSMC. Loss of p53 activity results in the growth of VSMC, while increased concentrations of p53 result in apoptosis of VSMC. A common polymorphism in the p53 amino acid sequence which results in the presence of either arginine (Arg) or proline (Pro) at position 72 may influence the susceptibility to malignancy through its interaction with p73. The effects of this polymorphism on p53 function seem to be related to p73, and p53Arg was reported to be more susceptible to the inactivation of p73 than p53Pro alleles. ${ }^{2}$

It is conceivable that this common polymorphism of p53, Arg72Pro, may also influence VSMC proliferation after coronary stent implantation. In the present study, we tested this hypothesis in patients after coronary stent implantation using quantitative coronary angiography.

\section{METHODS}

The study population was selected from outpatients at the National Cardiovascular Center, Osaka, Japan, who underwent follow up coronary angiography after successful stent placement. This genetic study was approved by our institutional ethics committee and included 132 consecutive patients admitted between August and October 1999, from whom informed consent was obtained. Major adverse cardiac events (death, myocardial infarction, coronary artery bypass graft surgery, and repeat interventions) did not occur in any of the patients undergoing stent implantation, as assessed by follow up angiography.

Quantitative computer assisted angiographic measurements were performed on end diastolic frames using an automated edge detection system CMS (MEDIS Medical Imaging Systems, Leiden, The Netherlands). The minimal lumen diameter (MLD), reference lumen diameter (RLD), and per cent diameter stenosis were obtained using this system.

Genomic DNA was extracted from peripheral blood leucocytes. The p53 genotype was determined using the TaqMan system, which combines polymerase chain reaction amplification and detection in a single closed tube. The primers and probes were used for allelic discrimination of p53Arg72Pro polymorphism. The validity of the TaqMan system was confirmed using DNA samples of the three genotypes as confirmed by direct sequencing.
VSMC were prepared from the aorta of p53 knockout mice. Transient transfection of the p53 gene was performed using Lipofect Amin (Gibco BRL) according to the instructions of the manufacturer. VSMC were plated onto a 96 well multititre plate (10 000 cells/well) and cultured in Dulbecco's modified Eagle's medium (DMEM) and 10\% fetal bovine serum (FBS) for 24 hours. After deprivation of serum for 24 hours, VSMC were transfected with wild/mutant p53 plasmids $(\mathrm{n}=48)$. Transfected VSMC were cultured in DMEM and 2\% FBS for 48 hours. Forty eight hours after the transfection, the cell number was counted with a WST (water soluble tetrazolium salt) cell counting kit (Wako). The expression construct of p53 was purchased from Invitrogen (GeneStorm expression-ready human clones). p53 cDNA is expressed under the control of a cytomegalovirus promoter. p53Pro was made from p53Arg by in vitro mutagenesis. The sequences of both constructs were confirmed by direct sequencing.

\section{RESULTS}

The 132 stenting lesions were divided into three groups according to the p53 genotype (47 Arg/Arg, 64 Arg/Pro, and 21 Pro/Pro). These genotype frequencies were compatible with the Hardy-Weinberg equilibrium. There were no significant differences in RLD among the three genotypes (3.00 (0.39) mm $v 3.00(0.44) \mathrm{mm} v 3.05$ (0.35) mm). MLD did not significantly differ before $(0.46(0.47) \mathrm{mm} v 0.52$ $(0.51) \mathrm{mm} v 0.48(0.48) \mathrm{mm})$ or immediately after stent implantation $\left(\begin{array}{lllll}2.82 & (0.47) \mathrm{mm} & v & 2.74 & (0.58) \\ \mathrm{mm} & v & 2.79\end{array}\right.$ $(0.42) \mathrm{mm}$ ) (fig 1A). However, MLD was significantly smaller $\quad\left(\begin{array}{lllll}1.36 & (0.86) \mathrm{mm} & v & 1.83 & (0.90) \\ \mathrm{mm} & v & 2.01\end{array}\right.$ $(0.92) \mathrm{mm}, \mathrm{p}<0.005)$ and the per cent diameter stenosis was significantly greater $(55(27) \% v 41 \quad(26) \% v 34(25) \%$, $\mathrm{p}<0.005)$ in the Arg/Arg genotype than in the other groups at follow up (fig 1B).

To assess the determinants of MLD at follow up, a multiple regression analysis with a backward elimination was performed. The results revealed that MLD at follow up was determined $(r=0.531, \mathrm{p}<0.0001)$ by the $\mathrm{p} 53$ genotype $(p=0.001)$, RLD $(p<0.005)$, diabetes mellitus $(p<0.05)$, and MLD immediately after stent implantation $(\mathrm{p}<0.05)$.

To confirm the effect of the p53 genotype on VSMC we transfected Arg and Pro of p53 genes to aortic VSMC from p53 knockout mice. The cell count after transfection with p53Arg (25 423 (4022), $\mathrm{n}=48$ ) was greater than that after transfection with p53Pro $\left(\begin{array}{lll}18623 & (2538), \quad \mathrm{n}=48\end{array}\right)$ $(\mathrm{p}<0.0001)$.

Abbreviations: Arg, arginine; DMEM, Dulbecco's modified Eagle's medium; FBS, fetal bovine serum; MLD, minimal lumen diameter; Pro, proline; RLD, reference lumen diameter; VSMC, vascular smooth muscle cells 

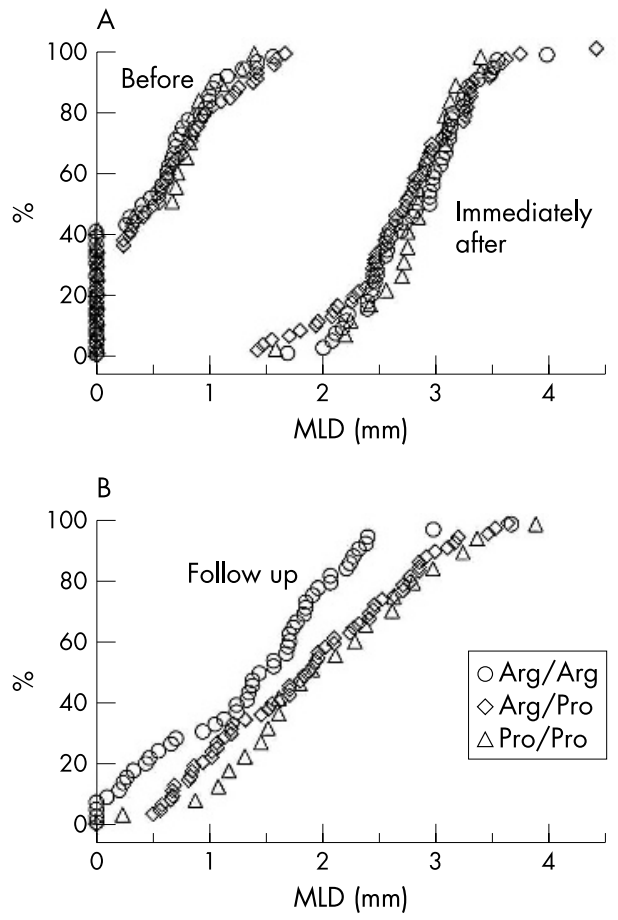

Figure 1 (A) Cumulative distribution curves of the MLD before and immediately after stent implantation for each genotype of $\mathrm{p} 53$. (B) Cumulative distribution curves of MLD at the time of follow up after stent implantation for each genotype of p53.

\section{DISCUSSION}

In the present study, we found that MLD at follow up angiography was significantly smaller in the Arg/Arg genotype than in the Arg/Pro and Pro/Pro genotypes of p53 in patients who underwent coronary stenting, and that MLD at follow up was determined by the p53 genotype, RLD, diabetes mellitus, and MLD immediately after stent implantation.

p53 has been shown to induce cell cycle arrest at the $G_{1} / S$ boundary and also apoptosis. Increased concentrations of p53 result in apoptosis of VSMC, while loss of p53 activity results in the growth of VSMC. Guevara and co-workers reported the excessive proliferation of VSMC at $S$ phase as a result of p53 inactivation in vivo. ${ }^{3}$ Taken together, the present results suggest that p53Arg72Pro polymorphism is related to the functional activity of $\mathrm{p} 53$, which regulates VSMC proliferation leading to luminal narrowing after stent placement.

We also demonstrated that p53Arg has less of an inhibitory effect on VSMC proliferation than p53Pro. The functional difference between p53 variants was observed in a recent study which showed that the p53Arg variant is more efficient than p53Pro at inducing apoptosis, and that one mechanism underlying this greater efficiency is enhanced localisation of the p53Arg variant to mitochondria. ${ }^{4}$ However, the localisation of p53 to mitochondria seems to occur only in tumour cells. ${ }^{5}$ Therefore, this p53Arg induced apoptosis is unlikely to be the cause of our findings. On the other hand, the manner of p53 according to the codon 72 polymorphic variants may be deeply affected by p73. Marin and colleagues ${ }^{2}$ recently reported that the Arg/Arg genotype in squamous cell tumours may reduce the inhibition of cell growth, possibly because the conformational p53 protein, consisting of the homozygote for p53Arg, can bind to the p73 protein, and neutralise p73induced apoptosis. This indicates that interaction of the p53 protein with the p73 protein is influenced by a common polymorphism of p53 at amino acid residue 72 . Consequently, VSMC proliferation may occur because p53Arg tends to block p73 function more effectively than p53Pro.

\section{ACKNOWLEDGEMENTS}

This work was supported in part by grants from the Japan Cardiovascular Research Foundation, the Fellows' Association of the Japanese Society of Internal Medicine, and the Ministry of Education, Culture, Sports, Science, and Technology. Also, this study was supported in part by the Program for Promotion of Fundamental Studies in Health Science for the Organization for Pharmaceutical Safety and Research of Japan.

\section{Authors' affiliations}

S Kojima, Department of Cardiovascular Medicine, Graduate School of Medical Sciences, Kumamoto University, Kumamoto City, Japan

N Iwai, N Tago, K Ono, Research Institute, National Cardiovascular Center, Osaka, Japan

K Ohmi, G Tsujimoto, Department of Pathology, National Children's Medical Research Center, Tokyo, Japan

S Takagi, S Miyazaki, H Nonogi, Y Goto, Division of Cardiology, Department of Medicine, National Cardiovascular Center, Osaka, Japan

Correspondence to: Dr Yoichi Goto, National Cardiovascular Center, 5-7-1, Fujishiro-dai, Suita, Osaka 565-8565, Japan; ygoto@ hsp.ncvc.go.jp

Accepted 24 March 2004

\section{REFERENCES}

1 Fattori R, Piva T. Drug-eluting stents in vascular intervention. Lancet 2003;361:247-9.

2 Marin MC, Jost CA, Brooks LA, et al. A common polymorphism acts as an intragenic modifier of mutant p53 behavior. Nat Genet 2000;25:47-54.

3 Guevara NV, Kim HS, Antonova El, et al. The absence of p53 accelerates atherosclerosis by increasing cell proliferation in vivo. Nat Med 1999;5:335-9.

4 Dumont $\mathrm{P}$, Leu JIJ, Pietra III ACD, et al. The codon 72 polymorphic variants of p53 have markedly different apoptotic potential. Nat Genet 2003;33:357-65

5 Marchenko ND, Zaika A, Moll UM. Death signal-induced localization of p53 protein to mitochondria. A potential role in apoptotic signaling. J Biol Chem 2000;275:16202-12. 